\title{
A Qualitative Simulation Approach for Fuzzy Dynamical Models
}

\author{
ANDREA BONARINI and GIANLUCA BONTEMPI \\ Politecnico di Milano
} This article deal with simulation of approximate models of dynamic systems. We propose an
approach that is appropriate when the uncertainty intrinsic in some models cannot be reduced
by traditional identification techniques, due to the impossibility of gathering experimental data
about the system itself. The article presents a methodology for qualitative modeling and
simulation of approximately known systems. The proposed solution is based on the Fuzzy Sets
theory, extending the power of traditional numerical-logical methods. We have implemented a
fuzzy simulator that integrates a fuzzy, qualitative approach and traditional, quantitative
methods.

Categories and Subject Descriptors: I.6.5 [Simulation and Modeling]: Model Development; I.6.7 [Simulation and Modeling]: Simulation Support Systems; I.6.8 [Simulation and Modelingl: Types of Simulation-combined

General Terms: Design, Experimentation, Theory

Additional Key Words and Phrases: Simulation of approximate dynamical models, simulation of fuzzy dynamical models

\section{INTRODUCTION}

Simulation can be considered as a part of the process of modeling and forecasting the behavior of a dynamic system. Its task is to reproduce, in the most suitable way, the evolution of a system model in time [Zeigler 1976].

A model is a finite set of formal relations that, in the traditional scientific approach, are mathematical relations among variables describing the real system. For some applications, it may be difficult to find a small set of elementary laws to describe the interesting aspects of the observed phenomenon: the model could be too complex for its effective simulation, or it could be difficult to identify its parameters. In these cases, the only available description, effectively usable in simulation, may be an approximate model affected by uncertainty. Therefore, the modeler should cope with uncertainty

This research was supported in part by the "Progetto Finalizzaro Informatica e Calcolo Parallelo" of the CNR objective "Ragionamento Qualitativo."

G. Bontempi's current address: IRIDIA, Univ. Libre de Bruxelles, Ave. Franklin Roosevelt 50 CP 19416, B-1050 Bruxelles, Belgium; email: gbonte@ulb.ac.be.

Permission to copy without fee all or part of this material is granted provided that the copies are not made or distributed for direct commercial advantage, ACM copyright notice and the title of the publication and its date appear, and notice is given that copying is by permission of ACM. To copy otherwise, or to republish, requires a fee and/or specific permission. (C) 1994 ACM 1049-3301/94/1000-0285 \$03.50 
and approximation if he or she wants to use the model to understand and forecast the behavior of a complex, real system.

The theory of stochastic parametric identification [Graupe 1972; Eykhoff 1974 ] is a well-known approach for working with approximate and uncertain system models, but it can only be applied when the system is accessible and measurable. In some cases a model of the system is available, but it is impossible to estimate with accuracy its parameters, either because we cannot observe the real system (e.g., a nuclear power plant in unexpected emergency situations) or because if does not yet exist (e.g., a production plant not yet built). It is useful to reason about models in these situations, too, even if the intrinsic uncertainty cannot be reduced by classical identification methods. Therefore, there is an interest in methods to encode and propagate uncertainty and approximation about the system.

In the last decade, research on qualitative modeling and simulation proposed many approaches to study incompletely known systems [Bobrow 1985; Weld and de Kleer 1990]. These were presented as alternatives to traditional, mathematical modeling and were based on mathematical-logical formalisms. However, the results are not completely satisfying: qualitative simulation does not always produce a univocal behavior, but only a tree of possible evolutions (e.g., Kuipers [1986]). Simulation of complex dynamic systems can produce an envisioning tree growing exponentially with the number of variables. This makes qualitative simulation computationally expensive when applied to complex systems [Milne 1991].

Moreover, in many engineering applications, a mathematical model could be available, although it may be impossible to identify its parameters precisely. In other words, there is a niche in the world of modeling applications, where both the traditional, numerical approach and the qualitative approach are not suitable: a numerical model is available, but it does not describe the system completely, which, in turn, is too complex for qualitative modeling.

We propose an approach to work in this niche. Our approach is based on the Fuzzy Set theory for implementing qualitative modeling and simulation. We consider the fuzzy formalism as an extension, not an alternative, to the classical, quantitative, mathematical formalism.

In this article we present the problems of a qualitative fuzzy simulation and our proposals to face them. We present the adopted methodology to design and implement the Qualitative Simulators, Qua.Si.I [Bonarini and Bontempi 1994a] and Qua.Si.II [Bonarini and Bontempi 1994b]. These simulators allow the description and simulation of approximate models, integrating fuzzy and traditional mathematics.

The simulators implement the following features:

(1) They accept ODE-based and algebraic models.

(2) They produce the same performances of a traditional numeric simulator if the model is precisely described in mathematical terms.

(3) They can simulate models where some or all of the initial conditions are approximately known.

ACM Transactions on Modeling and Computer Simulatıon, Vol 4, No 4, October 1994 
(4) They can simulate models where some or all of the parameters are approximately known.

(5) Approximation is modeled by fuzzy numbers [Dubois and Prade 1980].

(6) Qua.Si. I can also simulate systems modeled by fuzzy production rules.

We compare our approach with other proposals for fuzzy simulation [Fishwick 1990; Shen and Leitch 1993], putting in evidence the respective limitations. Finally, we present the results we obtained both on models of theoretical interest and on models used in real applications.

\section{THE QUA.Si. QUALITATIVE APPROACH}

In this section we introduce the key concepts and our extensions to fuzzy mathematics theory, on which the Qua.Si. simulation approaches are based.

\subsection{Fuzzy Sets and Numbers}

The notion of a fuzzy set is an extension of the concept of a boolean set. Let $X$ be a classical set of objects, called the universe, whose generic elements are denoted $x$. Membership in a boolean subset $A$ of $X$ can be viewed as a characteristic function, or membership function (m.f.)

$$
\begin{array}{ll}
\mu_{A}: X \rightarrow\{0,1\} & \text { such that } \\
\mu_{A}(x)=1 \quad \text { if } & x \in A, \\
\mu_{A}(x)=0 & \text { if } \quad x \notin A .
\end{array}
$$

$\{0,1\}$ is called the valuation set. If the valuation set is allowed to be the real interval $[0,1], A$ is called a fuzzy set.

Definition. An $\alpha$-cut of level $h(0 \leq h \leq 1)$ of a fuzzy set $A$ is the crisp set

$$
A_{h}=\left\{x \in A, \mu_{A}(x) \geq h\right\} .
$$

Definition. A fuzzy set A is normalized if $\exists x \mid \mu_{A}(x)=1$.

Definition. A fuzzy number is a convex, normalized fuzzy subset of the real domain $R$.

The concept of a fuzzy number is an extension of the notion of a real number: it encodes approximate quantitative knowledge [Kaufmann and Gupta 1985; Kandel 1986]. For instance, in a system (e.g., a tank) whose state (e.g., its internal pressure) is uncertain a fuzzy number (Figure 1) can formalize the qualitative knowledge: "the pressure inside the tank is approximately 5 bar."

\subsection{The Extension Principle and the $\alpha$-Cut Method}

The mathematics of fuzzy numbers is founded on the Extension Principle introduced by Zadeh [1965]. It provides a general method for extending standard mathematical concepts in order to deal with fuzzy quantities [Dubois and Prade 1980]. 
Fig. 1. A fuzzy number.

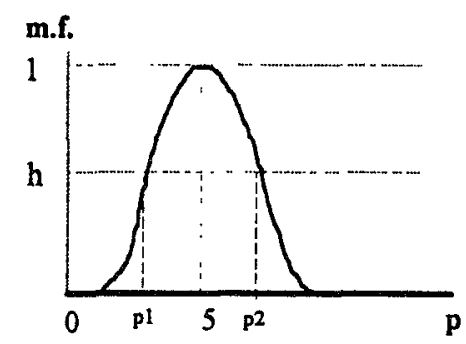

Let $\varphi: X 1 \times X 2 \times \cdots \times X n \rightarrow Y$ be a real mapping from $X 1 \times X 2 \times \cdots \times$ $X n$ to a universe $Y$ such that $y=\varphi\left(x_{1}, x_{2}, \ldots, x_{n}\right)$. The extension principle allows us to induce from $n$ fuzzy sets $\bar{x}_{\imath}$ on $X i$ a fuzzy set $\bar{y}$ on $Y$ through $\varphi$ such that

$$
\mu_{\bar{y}}(t)=\sup _{t=\varphi\left(s_{1}, s_{2}, \quad, s_{n}\right)} \min \left(\mu_{\overline{x_{1}}}\left(s_{1}\right), \mu_{\overline{x_{2}}}\left(s_{2}\right), \ldots, \mu_{\overline{x_{n}}}\left(s_{n}\right)\right)
$$

or

$$
\mu_{\bar{y}}(t)=0 \quad \text { if } \quad \varphi^{-1}(t)=\varnothing
$$

where $\varphi^{-1}(t)=0$ is the inverse image of $t$, and $\mu_{\overline{x_{t}}}$ is the m.f. of $\overline{x_{l}}(i=$ $1, \ldots, n)$.

The Extension Principle provides a method to compute the fuzzy value of a fuzzy mapping, but in practice, its application is not feasible because of the infinite number of computations it would require. However, some approaches to fuzzy mathematics have been proposed [Dubois and Prade 1979; Dong and Shah 1987; Baas and Kwakernaak 1977]. Unfortunately, each one of them introduces spurious values in the results.

Our approach considers fuzzy calculus as an extension of interval mathematics by the $\alpha$-cut notion. Nguyen [1978] proved that when cumputing the value of a fuzzy mapping a generic $h$-level $\alpha$-cut of the image depends only on the $h$-level $\alpha$-cuts of the arguments

$$
\overline{y_{h}}=\varphi\left(\overline{x_{1}}, \overline{x_{2}}, \ldots, \overline{x_{n}}\right)_{h}=\varphi\left(\overline{x_{1 h}}, \overline{x_{2 h}}, \ldots, \overline{x_{n h}}\right) .
$$

A fuzzy algebraic computation may be decomposed into several interval computations, each one having as arguments the $\alpha$-cut intervals of the fuzzy arguments at the same level. The results are the $\alpha$-cuts of the fuzzy result.

We call the method based on this $\alpha$-cut discretization of fuzzy arguments the $\alpha$-cut method. The fuzzy calculus problem is related to interval calculus. From now on, we will treat only interval computation.

\subsection{Interval Mathematics}

Moore [1966] dealt exhaustively with interval mathematics. Given two intervals $X=[x 1, x 2]$ and $Y=[y 1, y 2](x 1, x 2, y 1, y 2 \in R, x 1 \leq x 2$, and $y 1 \leq$ ACM Transactions on Modeling and Computer Simulation, Vol 4, No. 4, October 1994 
$y 2$, we denote interval mathematics as the mathematics based on the following arithmetic operations:

$$
\begin{gathered}
X+Y=[x 1+x 2, y 1+y 2] \\
X-Y=[x 1-y 2, x 2-y 1] \\
X^{*} Y=\left[\min \left(x 1^{*} y 1, x 2^{*} y 1, x 1^{*} y 2, x 2^{*} y 2\right),\right. \\
\left.\max \left(x 1^{*} y 1, x 2^{*} y 1, x 1^{*} y 2, x 2^{*} y 2\right)\right] \\
\frac{X}{Y}=[\min (x 1 / y 1, x 2 / y 1, x 1 / y 2, x 2 / y 2), \\
\max (x 1 / y 1, x 2 / y 1, x 1 / y 2, x 2 / y 2)] \quad \text { if } 0 \notin[y 1, y 2] .
\end{gathered}
$$

Given an interval $X=[x 1, x 2], W=x 2-x 1$ is said the width of $X$.

The following properties hold:

-Interval multiplication and addition are associative and commutative operations.

The distributive law does not always hold for interval arithmetic. It is not always valid that $I^{*}(J+K)=I^{*} J+I^{*} K$ for any $I, J$, and $K$.

-Inverses in multiplication and addition do not exist, in general.

In fact, for a generic $[a, b](a \leq b)$, an interval $[c, d]$ such that $[a, b]+[c, d]$ $=0\left([a, b]^{*}[c, d]=1\right.$ in the multiplication case) does not exist. Moreover, given the real rational mapping $f: R^{n} \rightarrow R$ the following relation holds [Moore 1966]:

$$
F(X 1, X 2, \ldots, X n) \supseteq f(X 1, X 2, \ldots, X n),
$$

where

$-f(X 1, X 2, \ldots, X n)$ is the set of values of the function $f(x 1, x 2, \ldots, x n)$ when its arguments range over the interval arguments $X 1, X 2, \ldots, X n$.

$-f(X 1, X 2, \ldots, X n)=\{y \mid y=f(z 1, z 2, \ldots, z n), z 1 \in X 1, z 2 \in X 2, \ldots, z n \in$ $X n\}$.

$-F(X 1, X 2, \ldots, X n)$ is the interval value of the function $f(x 1, x 2, \ldots, x n)$ computed by using interval arithmetic and by assigning $x 1=X 1, \ldots, x n$ $=X n$.

$f$ is a function with interval arguments, while $F$ is an interval function.

Therefore, if $(x 1, x 2, \ldots, x n)$ is a set of state variables of a real system, according to Eq. (4), $f(X 1, X 2, \ldots, X n)$ models consistently the approximated relation $f$ existing between the variables $(x 1, x 2, \ldots, x n)$, whereas interval arithmetic may introduce spurious values in the solution. In other words, $F(X 1, X 2, \ldots, X n)$ is not the correct solution from the modeler's point of view.

\subsection{Interaction and Interval Calculus}

The correct solution of a single function with interval arguments can come only from a numerical analysis of the function, able to locate maximum and 


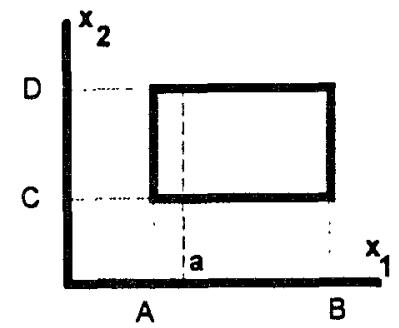

(a)

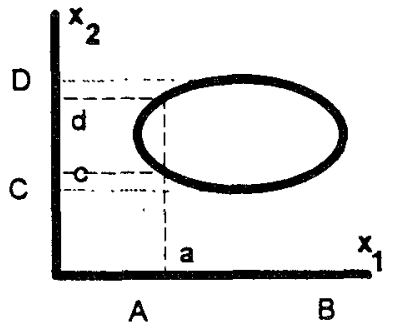

(b)

Fig. 2. Relation of interaction between intervals $I_{1}=[\mathrm{A}, \mathrm{B}]$ and $I_{2}=[\mathrm{C}, \mathrm{D}]$. (a) noninteracting. (b) Interacting.

minimum points (we call them extreme points) inside of the argument domains: if we compute the function for these points, we may obtain the correct solution for a single relation. However, if a model has more algebraic relations whose arguments are correlated, the method of extreme points does not guarantee the correct solution. This is due to the interaction between variables.

Two variables, whose values are, respectively, the two intervals $I_{1}=[\mathrm{A}, \mathrm{B}]$ and $I_{2}=[\mathrm{C}, \mathrm{D}]$, are said to be noninteracting if there is no relation between the values they can take into their domains. In other words (Figure 2), if $x_{1}=a \in I_{1}, x_{2}$ is not bound to any specific value into $I_{2}$. In the opposite case, they are said to be interacting, since $x_{1}=a$ implies that $x_{2}$ ranges over a specific interval $[c, d]$ contained in $I_{2}$. Then, intervals $I_{1}$ and $I_{2}$ do not represent univocally the approximate relationship existing between the variables $x_{1}$ and $x_{2}$.

As a consequence, any method able to compute the correct range of a function $f(X 1, X 2, \ldots, X n)$ of intervals may be useless if its arguments are interacting. Considering interacting variables as noninteracting introduces spurious values into the result of the computation. As an example, consider a system whose behavior at time $t$ is described by the following relations:

$$
\begin{aligned}
& y(t)=x_{1}(t)^{2}-2 x_{2}(t) \\
& z(t)=y(t)+2 x_{2}(t) .
\end{aligned}
$$

The initial conditions are the intervals $x_{1}=[-1,1]$ and $x_{2}=[2,3]$. Using the method of the extreme points, we have

$$
y(t)=[-6,-3]
$$

after the calculus of (5), and using the result in (6),

$$
z(t)=[-2,3]
$$

after the calculus of (6).

Nevertheless, even applying the method of extreme points the result is not correct because of the interaction between $x_{2}$ and $y$, by relation (5). The correct result is

$$
z(t)=[0,1],
$$


as we can see by making a substitution of (5) in (6), which yields

$$
z(t)=x_{1}(t)^{2} .
$$

Now consider the following. Every algebraic computation with arguments represented as intervals (or fuzzy numbers considered as $\alpha$-cut collections) is not correct if some of its arguments are interacting. This is not due to the resolution method, but to the interval (and fuzzy) formalism, which cannot represent interaction and which is responsible for the introduction of spurious values in any algebraic computation.

Any fuzzy computation with several algebraic relations may be affected by errors: it provides a result wider than or equal to the correct one. Then, in a fuzzy computation with several algebraic relations, it is useless to waste computational time in the computation of the single parts by the method of extreme points, because their connection is inevitably affected by errors. Moreover, no computation based on the method of extreme points can be performed in a time convenient for a simulation.

Therefore, in the first version of Qua.Si. we did not take that choice, and we implemented the calculus of any fuzzy algebraic relation by the $\alpha$-cut method based on interval mathematics. Despite the errors, it always provides a result containing or equal to the exact one, according to Eq. (4).

\subsection{Differential Calculus and Fuzzy Numbers}

We now consider fuzzy calculus as a method for the simulation of differential equations with fuzzy parameters and/or fuzzy variables. Again, we point out the correspondence between this problem and the analogous one for interval mathematics, thanks to the $\alpha$-cut notion.

Consider the initial value problem in ordinary differential equations (ODE). Under very general conditions, an ODE of any finite order, or a system of differential equations of various finite orders, can be put into the form of an autonomous system of first-order equations

$$
\frac{d x_{l}(t)}{d t}=f_{i}\left(x_{1}(t), \ldots, x_{n}(t)\right), \quad i=1,2, \ldots, n .
$$

Using vector notation we can give a more concise representation of (7):

$$
\frac{d x(t)}{d t}=f(x(t)) .
$$

A vector function $f: E^{n} \rightarrow E^{n}$ on an Euclidean $n$-dimensional vector space $E^{n}$ is said to define a vector field on $E^{n}$ (phase space).

Under given conditions on the function $f$, the vector differential Eq. (8) is said to define a flow or a dynamical system on the phase space $S$. Through each point $\bar{x}$ in $S$, when $t=\bar{t}$, a unique solution $x(t, \bar{x})$ lying in $S$ passes with $x(\bar{t}, \bar{x})=x$.

In the nonapproximate case, $x(0)=x_{0}$, where $x_{0}$ is a vector of real numbers that are the certain initial conditions of the system. 
Assume that the functions $f_{1}(x)=f_{1}\left(x_{1}, x_{2}, \ldots, x_{n}\right), i=1,2, \ldots, n$, have continuous total derivatives of all orders with respect to $t$ along solutions to (7) for all points in some open set in $E^{n}$ containing the initial point $x_{0}$. That is, we define $f_{1}^{(J)}(x 1(t), \ldots, x n(t))$ recursively by

$$
f_{l}^{(0)}\left(x_{1}(t), \ldots, x_{n}(t)\right)=f_{l}\left(x_{1}(t), \ldots, x_{n}(t)\right)
$$

and

$$
f_{t}^{(j)}=\sum_{m=1}^{n} \frac{\partial f_{l}^{(J-1)}}{\partial x_{m}} f_{m}^{(0)} \quad \text { for } \quad j=1,2, \ldots
$$

where

$$
f_{l}^{(J)}\left(x_{1}(t), \ldots, x_{n}(t)\right) \text { denotes } f_{l}^{(J)} .
$$

If the functions $x_{1}(t), \ldots, x_{n}(t)$ have continuous derivatives of all orders for $t \in[0, h]$, then the Taylor theorem with remainder asserts that for $t \in[0, h]$, $k>1$,

$$
\begin{aligned}
x_{i}(t)=x_{i}(0) & +\sum_{j=1}^{k-1}\left(\frac{f_{l}^{(j-1)}\left(x_{1}(0), \ldots, x_{n}(0)\right)}{j !}\right) t^{j} \\
& +\frac{f_{i}^{(k-1)}\left(x_{1}(a), \ldots, x_{n}(a)\right)}{k !} t^{k}
\end{aligned}
$$

for some $a \in[0, t]$. The coefficients of the Taylor formula for a numeric differential system are numeric coefficients.

Assume now that the initial condition is uncertain and that it is represented by an interval vector $X(0)$. Extending the Taylor formula to intervals, $x(t)$ has the value $X(t)$ for a generic $t$ whose $i$ th component is

$$
X_{\imath}(t) \cong X_{l}(0)+\sum_{j=1}^{(k-1)} \frac{f_{i}^{(j-1)}(X(0))}{j !} t^{j} .
$$

The most common numerical integration routines for ODE resolution implement an approximation to Taylor series. If parameters or initial conditions are intervals as in Eq. (11), Taylor's formula becomes an algebraic sum of addenda, each one of which takes an interval value. In a sum computed by interval arithmetic, the width of the result is always equal to the sum of the widths of the two addenda. As a result, if we used interval arithmetic we would obtain an interval value of $x$, which becomes wider with $t$ increasing. In the case of a dynamic system, where the independent variable $t$ is the time, the system output would be affected by a width always increasing, independently of stability or other properties of the system. It could happen that the evolution of the system reaches regions of the phase space, where no numerical solution of the differential system passes through. Thus, the system simulation based on interval mathematics would produce outputs in contradiction to the physical reality described by the model. Therefore, when applying interval mathematics to Taylor series, undesired errors are introduced. 
Later, in Sections 3 and 4, we present our two alternative approaches for the simulation of ODEs where parameters and/or initial conditions are interval valued.

\subsection{On the Simulation of ODEs where Parameters and / or Initial Conditions Are Interval Valued}

Consider a system of ODEs in a vectorial form where variable and parameter values can be expressed as intervals. $n$ is the order of the system, and $k$ is the number of interval-valued parameters. Then

$$
d X(t) / d x=f(X(t), P),
$$

where

$$
X(0)=X_{0}=\left[X_{01}, X_{02}, \ldots, X_{0 n}\right]
$$

is the vector of initial interval conditions and where

$$
P=\left[P_{1}, P_{2}, \ldots, P_{K}\right]
$$

is the vector of parameters whose value is an interval.

The interval values of the $N=n+k$ entities generate an $N$-cube at $t=0$; every point of this hypercube is the initial condition of a solution of the differential equation system. We call this $N$-cube the region of uncertainty of the system at $t=0$. The complete ODE correct solution could be obtained by repeating a numerical integration starting from any point of the $N$-cube, but this is impossible in a continuous domain. Ranging the values of the variables over the $n$ intervals $X_{0}$, the ODE solution changes because of initial condition variation, whereas ranging the values of the parameters over the $k$ intervals $P_{y}$, the ODE form itself changes.

We maintain that, under general conditions, it is sufficient to repeat the computation for any point of the external surface of the $N$-cube ${ }^{1}$ at time $t$ to obtain the spatial distribution of the $N$-cube at the next time point. Let us transform the system of order $n$ and $k$ parameters in an equivalent system of order $N=n+k$, adding for each parameter $P_{J}$ the differential equation

$$
P_{J}^{\prime}=0, \quad j=1, \ldots, k .
$$

These equations hold for any $t$, since parameters are constant entities. We now have $N=n+k$ state variables and no interval parameters.

Let us define an $N$-dimensional state vector $Z$ such that

$$
\begin{array}{lll}
Z_{\imath}=Y_{\imath} & \text { for } & i=1, \ldots, n \\
Z_{\imath}=P_{\imath-n} & \text { for } & i=n+1, \ldots, N .
\end{array}
$$

ODE (12) becomes

$$
d Z(t) / d t=G(Z(t))
$$

of $N$ th order.

\footnotetext{
${ }^{1}$ The region of uncertainty at time $t>0$ is not a polyhedron anymore. We still denote it as an $N$-cube to suggest its $N$ th-order dimension.
} 


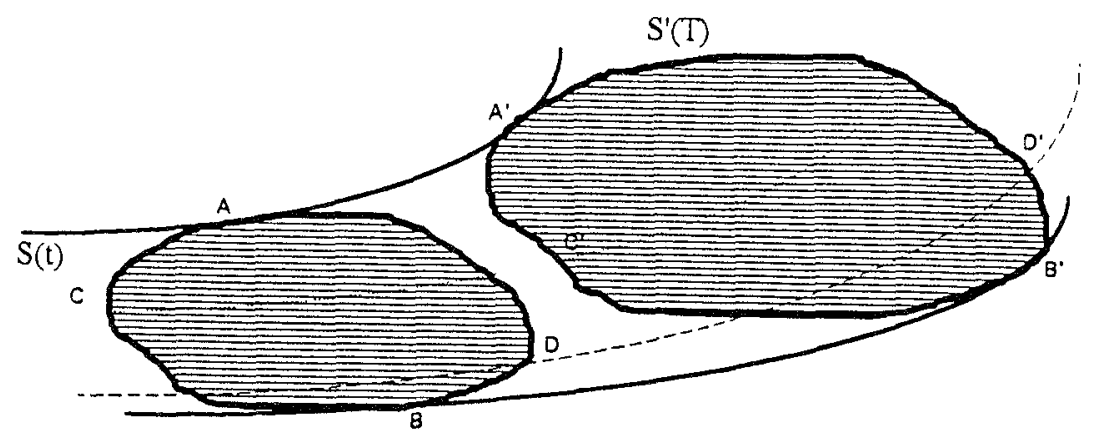

Fig. 3. Evolution of the external surface of the region of uncertainty.

We demonstrate that for any time $t$ the external surface of the $N$-cube depends only on the external surface of the $N$-cube at the previous instant. Let $S(t)$ be the external surface delimiting the $N$-cube at instant $t$. Then

$$
\begin{aligned}
& Z(0)=\text { initial } N \text {-cube } \\
& S(0)=\text { external surface of } Z(0) \text { at time } t=0 .
\end{aligned}
$$

Consider a time interval $[t, t+d t]$. Let us call $S^{\prime}(T)$ the surface in a generic time $T>t$ and $T \leq t+d t$, obtained by mapping the surface $S(t)$ according to ODE (14) (Figure 3). The following theorem holds:

THEOREM 2.6.1. An ODE maps the external surface of its region of uncertainty at time $t$, into the external surface of its region of uncertainty at time $t+d t$; that is, $S(t+d t)=S^{\prime}(t+d t)$.

Proof. Assume, $a b$ absurdo, that at time $t+d t$ a point on the external surface of the region of uncertainty $S(t+d t)$ is the mapping of a point $Q$ that was at time $t$ inside the external surface of the region of uncertainty $S(t)$. Therefore, $Q(t)$ is inside $S(t)$, while $Q(t+d t)$ belongs to $S(t+d t)$.

Then, there is a time $t^{*}$ such that $t<t^{*}<t+d t$, when the surface $S^{\prime}\left(t^{*}\right)$ (mapping of $S(t)$ at time $t^{*}$ ) is crossed by the trajectory of $Q$ : as a consequence it belongs to $S\left(t^{*}\right)$. There is a point $X \equiv Q\left(t^{*}\right)$ in the phase space where two trajectories of the ODE pass through at the same time $t^{*}$ (Figure 4):

(1) the trajectory of $Q$; and

(2) the trajectory of $P \in S(t)(P(t) \neq Q(t))$, since $S^{\prime}\left(t^{*}\right)$ is the geometric locus at time $t^{*}$ of points belonging to $S(t)$ at time $t$.

This is in contradiction to the solution of the Cauchy problem. It follows that the initial hypothesis was wrong. No points that are mappings of points interior to $S(t)$ at time $t$ belong to $S(t+d t)$. Thus, $S(t+d t)$ coincides with $S^{\prime}(t+d t)$.

The validity of the theorem leads to the conclusion that it is sufficient to calculate the trajectories of the points belonging to the external surface of the 


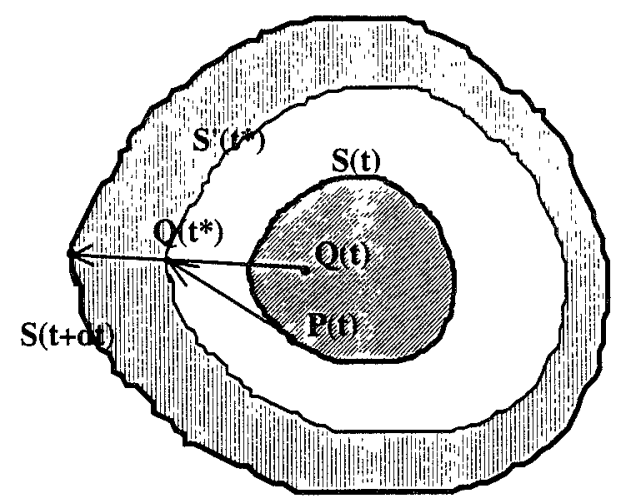

Fig. 4. $Q\left(t^{*}\right)$ is the intersection of two trajectories in the second order phase space of the ODE, in contradiction to the solution of the Cauchy problem.

region of uncertainty to know the evolution in time of the region itself. However, the quantity of trajectories to be computed is still infinite, even if it is of a lower order. The problem, now, is how to sample, in the most convenient way, the external surface of a hypercube during its time evolution in the phase space.

\section{THE NONINTERACTING APPROACH}

In the first version of our qualitative simulator (Qua.Si. I), we have implemented an algorithm that uses some of these points and that can be extended to work with an arbitrary number of points. It consists of the repetition of these steps:

- sampling of the $N$-cube external surface at the instant $t ;{ }^{2}$

-numeric integration for any sampled point until the instant $t+\Delta t$; and

${ }^{2}$ The number of steps in this operation is controlled by a parameter of recursion, called sampling index IS. In an $N$-cube there are $\left(\begin{array}{c}N \\ i\end{array}\right) * 2^{(N-1)}$ hyperfaces of $i$ th $\operatorname{dimension}(i=1, \ldots, N)$; i.e., for $N=3$ there are 6 faces of dimension 2 (rectangles) and 12 faces of dimension 1 (edges). The sampling algorithm follows these steps:

(1) Consider the $2^{N}$ vertices of the $N$-cube as sampling points.

(2) For any face of $j$ th $(j=1, \ldots, N-1)$ dimension, consider the geometric centers as sampling points, and divide the face in $2^{J}$ equal subfaces $S_{J, 2}$. The number of points considered is now $3^{N}-2^{N}-1$.

Continuing for any $s \leftarrow I S-1$ we have the following generic step:

(s) For any subface $S_{j, s-1}$ of $j$ th dimension $(j=1, \ldots, N-1)$, consider the geometric centers, and divide the subface in $2^{j}$ equal subfaces $S_{j, s}$. The number of points considered is

$$
\sum_{j=1}^{N-1}\left(\begin{array}{c}
N \\
j
\end{array}\right) * 2^{(N-J)} *\left(2^{s-2}\right)^{j}
$$

When the index of sampling is $I S$, the total number of points considered is

$$
2^{N}+\sum_{s=2}^{I S}\left(\sum_{j=1}^{N-1}\left(\begin{array}{l}
N \\
j
\end{array}\right) * 2^{(N-\jmath)} *\left(2^{s-2}\right)^{J}\right) .
$$


Fig. 5. Noninteracting approach makes the region of uncertainty noninteracting and introduces spurious points (black regions).

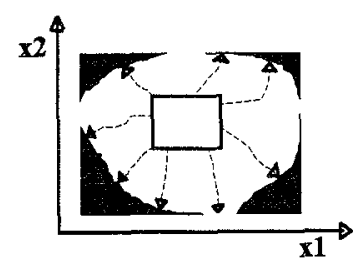

-reconstruction of the noninteracting $N$-cube at the instant $t+\Delta t$. The approximation of any variable at time $t+\Delta t$ is given by the interval ranging from the minimum to the maximum value obtained in the previous integration step.

This procedure is repeated for any $\alpha$-cut level considered in the fuzzy values discretization, thus obtaining the fuzzy evolution of the dynamic system.

This resolution of ODE, having intervals as initial conditions, considers the variables as always noninteracting. At the end of any integration step, the $N$-cube is reconstructed. This implies an important drawback: the insertion of spurious trajectories that do not belong to the system behavior.

At the beginning, all variables are noninteracting, but the computation leads inevitably to an interaction that has to be eliminated at each step in order to proceed in a noninteracting way. Figure 5 shows an interval ODE of order 2. The inner rectangle is the state space at the initial instant when the variables $x 1$ and $x 2$ are not interacting. The outer irregular figure is the state space at the next instant. In a noninteracting approach, it is necessary to approximate the state space with an $N$-cube. This inevitably adds some spurious areas to the correct points (the black regions in the figure), which are nonphysical.

An example is the simulation of the following differential system:

$$
\left\{\begin{array}{l}
\dot{x}=y \\
\dot{y}=-x
\end{array}\right.
$$

The solution is a sinusoidal function of the time (Figure 6). The initial conditions are the intervals $x(0)=[-1,1], y(0)=[3,4]$. In the state space, the initial uncertainty region is the rectangle ABCD. The differential relation maps it into $A^{\prime} B^{\prime} \mathrm{C}^{\prime} \mathrm{D}^{\prime}$ at time $t+\Delta t$. Then, the noninteracting algorithm makes the variables noninteracting and transforms $\mathrm{A}^{\prime} \mathrm{B}^{\prime} \mathrm{C}^{\prime} \mathrm{D}^{\prime}$ into the 2-cube FGHI. This 2-cube contains a set of points not belonging to the solution (black regions). They will be taken into account in the next step and will add spurious sinusoidal trajectories with a greater width $\left(\mathrm{HH}^{\prime}, \mathrm{II}^{\prime}\right)$. As a result, we have an output whose width is continuously increasing (see Figure 12).

The noninteracting algorithm does not introduce errors during the integration step, but it is forced to do so when it uses the results of the integrations. In fact, the interaction created during the integration step is lost because the interval or fuzzy formalism cannot represent it. To solve this problem we present a second approach based on the concept of the connection matrix. 


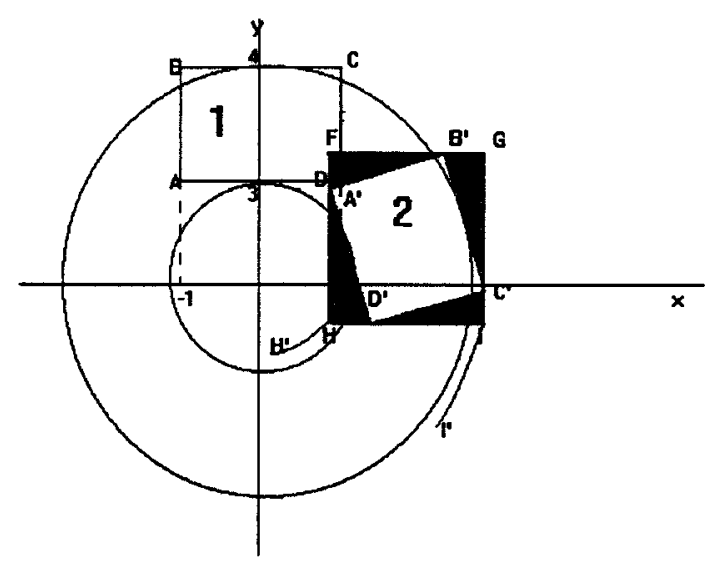

Fig. 6. Introduction of spurious trajectories in the noninteracting simulation of an oscillating system.

\section{THE INTERACTING APPROACH}

We denote by $x\left(t, x_{0}\right)$ the $n$-dimensional, real vector solution to the numeric initial-value problem

$$
\frac{d x(t)}{d t}=f(x(t)), \quad x(0)=x_{0} .
$$

We define the connection matrix for the solution $x\left(t, x_{0}\right)$ as the matrix $C\left(t, x_{0}\right)$ with elements

$$
c_{i J}\left(t, x_{0}\right)=\left.\frac{\partial x_{\imath}(t, \bar{x})}{\partial \bar{x}_{j}}\right|_{\bar{x}=x_{0}} .
$$

We denote by $J\left(t, x_{0}\right)$ the Jacobian matrix of the vector function $f$ evaluated at $x\left(t, x_{0}\right)$. The matrix $J\left(t, x_{0}\right)$ has elements

$$
J_{\imath j}\left(t, x_{0}\right)=\left.\frac{\partial f_{l}(x)}{\partial x_{J}}\right|_{x=x\left(t, x_{0}\right)} .
$$

Moore [1966] demonstrated that

$$
\frac{\partial C\left(t, x_{0}\right)}{\partial t}=J\left(t, x_{0}\right) C\left(t, x_{0}\right)
$$

with $C\left(0, x_{0}\right)=I$, where $I$ is the identity matrix.

The elements of the connection matrix give the sensitivity of the solution components $x_{i}\left(t, x_{0}\right)$, with respect to small changes of the initial values $\left(x_{o f}\right)$, with $j=1,2, \ldots, n$. If $x_{0}$ denotes a geometric point that belongs to the external surface of the initial uncertainty region of an interval ODE, coefficients $c_{i j}$ have a special meaning. With their values we can compute how the directions of the tangents to the uncertainty region change in time.

ACM Transactions on Modeling and Computer Simulation, Vol. 4, No. 4, October 1994. 


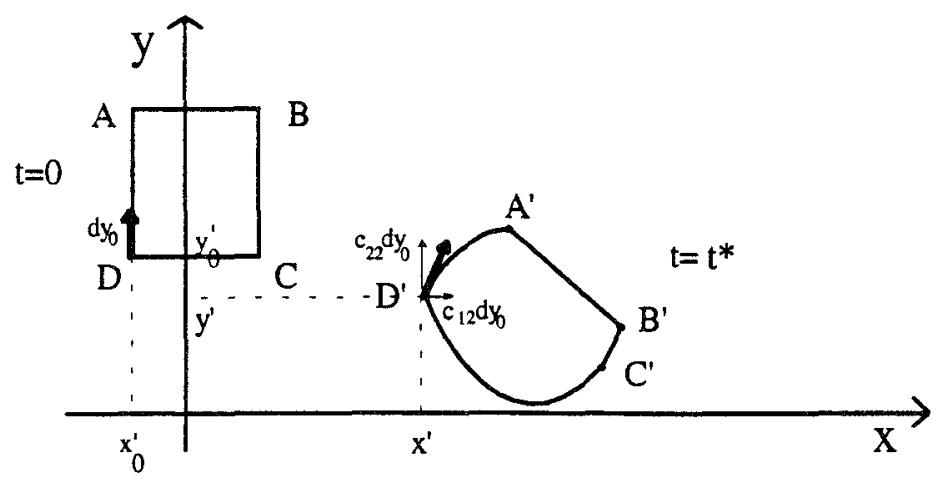

Fig. 7. Evolution of the external surface of the region of uncertainty for a second-order system.

Consider an ODE of second order whose generic trajectory in 2-D space is described by the following equations at time $t^{*}$ :

$$
\begin{aligned}
& x=x\left(x_{0}, y_{0}, t^{*}\right) \\
& y=y\left(x_{0}, y_{0}, t^{*}\right) .
\end{aligned}
$$

$\left(x^{\prime}, y^{\prime}\right)$ is the point in the 2-D phase space, reached at time $t^{*}$ by the trajectory starting in $\left(x_{0}^{\prime}, y_{0}^{\prime}\right)$ (Figure 7 ). The surface of the uncertainty region is a function of the parameters $x_{0}, y_{0}$. The $c_{l_{J}}$ coefficients are the partial derivatives with respect to these parameters. The coordinates of $D$ are $\left(x_{0}^{\prime}, y_{0}^{\prime}\right)$, and those of $D^{\prime}$ are $\left(x^{\prime}, y^{\prime}\right)$.

Compute the connection matrix associated with $\left(x^{\prime}, y^{\prime}, t^{*}\right)$ :

$$
C\left(x^{\prime}, y^{\prime}, t^{*}\right)=\left|\begin{array}{ll}
\frac{\partial x\left(x_{0}^{\prime}, y_{0}^{\prime}, t^{*}\right)}{\partial x_{0}} & \frac{\partial x\left(x_{0}^{\prime}, y_{0}^{\prime}, t^{*}\right)}{\partial y_{0}} \\
\frac{\partial y\left(x_{0}^{\prime}, y_{0}^{\prime}, t^{*}\right)}{\partial x_{0}} & \frac{\partial y\left(x_{0}^{\prime}, y_{0}^{\prime}, t^{*}\right)}{\partial y_{0}}
\end{array}\right|=\left|\begin{array}{ll}
c_{11} & c_{12} \\
c_{21} & c_{22}
\end{array}\right| .
$$

From Figure 7 we can see that an infinitesimal move $d y_{0}$ of the point $D$ along the external surface of the initial uncertainty region corresponds to an infinitesimal move of the point $D^{\prime}$ along the external surface of the uncertainty region at time $t^{*}$. Its Cartesian components are $d x^{\prime}=c_{12} d y_{0}^{*}$ and $d y^{\prime}=c_{22} d y_{0}^{*}$ with $c_{12}$ and $c_{22}$ coefficients of $C\left(x^{\prime}, y^{\prime}, t^{*}\right)$. As will be shown, we will use the connection matrix for a more convenient sampling of the external surface of the region of uncertainty.

\subsection{Property of Sufficiency of Vertices}

Consider a generic parametric line $L$ in $3-\mathrm{D}$ space (Figure 8 ) whose equations are

$$
\begin{aligned}
& x=x(v), \\
& y=y(v), \\
& z=z(v), \quad \text { with } \quad v \in V \equiv\left[v_{0}, v_{1}\right] .
\end{aligned}
$$




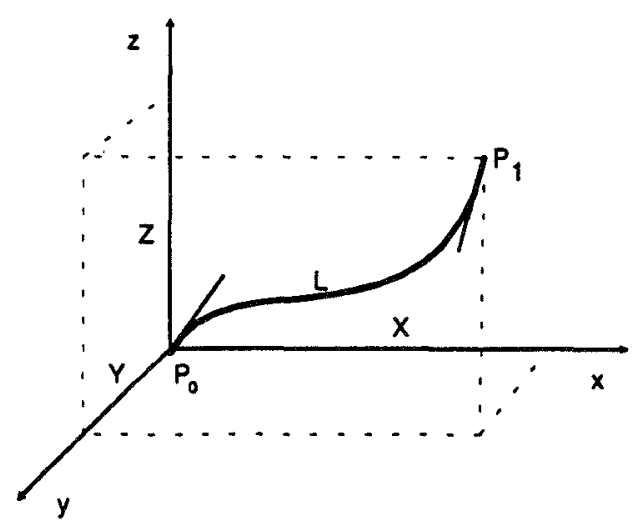

Fig. 8. Parametric line in 3-D space.

When $v$ ranges over the interval $V, x, y$, and $z$ range, respectively, over the intervals $X, Y$, and $Z$. How can we compute $X, Y$, and $Z$ ?

If $x(v), y(v)$ and $z(v)$ are monotonous functions of $v$, it is sufficient to know the coordinates $(x(v 0), y(v 0), z(v 0))$ and $(x(v 1), y(v 1), z(v 1))$ of the extremes $P_{0}$ and $P_{1}$ of $L$ to compute $X, Y$, and $Z$. Moreover, in general, given a line $L$ defined into an $n$-dimensional space whose equations are

$$
x_{\imath}=x_{i}(v), \quad v \in\left[v_{0}, v_{1}\right], \quad i=1,2, \ldots, n,
$$

we say that $L$ satisfies the property of sufficiency of the vertices (PSV) if it is sufficient to know the values of $L$ at the extremes $P_{0}$ and $P_{1}$ to compute the intervals $X_{L}$ such that

$$
x_{\imath}(k) \in X_{\imath}, \quad \forall k \in\left[v_{0}, v_{1}\right], \quad i=1,2, \ldots, n .
$$

The following necessary condition for the PSV holds. For any pair of points $P_{0 k} \in L_{v 0}$ (part of the line $L$ corresponding to a right neighborhood of $v_{0}$ ) and $P_{1} \in L_{v 1}$ (part of the line $L$ corresponding to a left neighborhood of $v_{1}$ ), the total derivatives with respect to $v$ have the same sign, that is,

$$
\begin{aligned}
& \frac{\partial x}{\partial v}\left(P_{1 \imath}\right) * \frac{\partial x}{\partial v}\left(P_{0 k}\right)>0 \\
& \frac{\partial y}{\partial v}\left(P_{1 i}\right) * \frac{\partial y}{\partial v}\left(P_{0 k}\right)>0 \\
& \frac{\partial z}{\partial v}\left(P_{1 \imath}\right) * \frac{\partial z}{\partial v}\left(P_{0 k}\right)>0 .
\end{aligned}
$$

As a consequence, the fact that the relations (18) hold only for $P_{1 \imath}=P_{1}$ and $P_{0 k}=P_{0}$ is still a necessary condition for the PSV. In fact, the PSV implies that relations (18) hold,

$$
\forall\left(P_{0 k}, P_{1 J}\right) \mid P_{0 k} \in L_{v 0} \quad \text { and } \quad P_{1_{J}} \in L_{v 1},
$$

which, in turn, implies that relations (18) hold,

$$
\forall\left(P_{0 k}, P_{1 j}\right) \mid\left(P_{0 k}=P_{0} \text { and } P_{1 j}=P_{1}\right) .
$$


Let us extend this notion to a surface $S$ of $j$ th order $(0<j<n)$ defined into an $n$-dimensional space by

$$
x_{l}=x_{\imath}\left(v_{1}, v_{2}, \ldots, v_{j}\right), \quad v_{J} \in\left[v_{0_{J}}, v_{1_{J}}\right] .
$$

We say that $S$ satisfies the PSV if it is sufficient to know the values of $S$ at its $2^{J}$ extremes to compute the intervals $X_{\imath}$ such that

$$
x_{l}\left(k_{1}, k_{2}, \ldots, k_{J}\right) \in X_{l}, \quad \forall k_{J} \in\left[v_{0_{J}}, v_{1 \jmath}\right], \quad i=1,2, \ldots, n .
$$

A necessary condition for the PSV is

$$
\frac{\partial x_{i}}{\partial v_{h}}\left(P_{1}\right) * \frac{\partial x_{\imath}}{\partial v_{h}}\left(P_{2}\right) * \cdots * \frac{\partial x_{\imath}}{\partial v_{h}}\left(P_{2 j}\right)>0, \quad i=1, \ldots, n, \quad h=1, \ldots, j .
$$

Now consider the application of these results.

We have, for instance, a third-order ODE system. Its initial uncertainty region is a 3 -cube delimited by the vertices $\mathrm{A}, \mathrm{B}, \mathrm{C}, \mathrm{D}, \mathrm{E}, \mathrm{F}, \mathrm{G}$, and $\mathrm{H}$ (Figure 9). Let us consider only a portion of the surface of the 3-cube, for instance, the face $\mathrm{ABCD}$. The $\mathrm{ODE}$ evolution transforms $\mathrm{ABCD}$ into $\mathrm{A}^{\prime} \mathrm{B}^{\prime} \mathrm{C}^{\prime} \mathrm{D}^{\prime}$, whose parameters are $x_{0}$ and $z_{0}$ ( $y_{0}$ is constant on $\mathrm{ABCD}$ ). A necessary condition for $\mathrm{A}^{\prime} \mathrm{B}^{\prime} \mathrm{C}^{\prime} \mathrm{D}^{\prime}$ to satisfy the $\mathrm{PSV}$ is that the following relations hold:

$$
\begin{aligned}
& \frac{\partial x}{\partial x_{0}}\left(\mathrm{~A}^{\prime}\right) * \frac{\partial x}{\partial x_{0}}\left(\mathrm{~B}^{\prime}\right) * \frac{\partial x}{\partial x_{0}}\left(\mathrm{C}^{\prime}\right) * \frac{\partial x}{\partial x_{0}}\left(\mathrm{D}^{\prime}\right)>0 \\
& \frac{\partial y}{\partial x_{0}}\left(\mathrm{~A}^{\prime}\right) * \frac{\partial y}{\partial x_{0}}\left(\mathrm{~B}^{\prime}\right) * \frac{\partial y}{\partial x_{0}}\left(\mathrm{C}^{\prime}\right) * \frac{\partial y}{\partial x_{0}}\left(\mathrm{D}^{\prime}\right)>0 \\
& \frac{\partial z}{\partial x_{0}}\left(\mathrm{~A}^{\prime}\right) * \frac{\partial z}{\partial x_{0}}\left(\mathrm{~B}^{\prime}\right) * \frac{\partial z}{\partial x_{0}}\left(\mathrm{C}^{\prime}\right) * \frac{\partial z}{\partial x_{0}}\left(\mathrm{D}^{\prime}\right)>0 \\
& \frac{\partial x}{\partial z_{0}}\left(\mathrm{~A}^{\prime}\right) * \frac{\partial x}{\partial z_{0}}\left(\mathrm{~B}^{\prime}\right) * \frac{\partial x}{\partial z_{0}}\left(\mathrm{C}^{\prime}\right) * \frac{\partial x}{\partial z_{0}}\left(\mathrm{D}^{\prime}\right)>0 \\
& \frac{\partial y}{\partial z_{0}}\left(\mathrm{~A}^{\prime}\right) * \frac{\partial y}{\partial z_{0}}\left(\mathrm{~B}^{\prime}\right) * \frac{\partial y}{\partial z_{0}}\left(\mathrm{C}^{\prime}\right) * \frac{\partial y}{\partial z_{0}}\left(\mathrm{D}^{\prime}\right)>0 \\
& \frac{\partial z}{\partial z_{0}}\left(\mathrm{~A}^{\prime}\right) * \frac{\partial z}{\partial z_{0}}\left(\mathrm{~B}^{\prime}\right) * \frac{\partial z}{\partial z_{0}}\left(\mathrm{C}^{\prime}\right) * \frac{\partial z}{\partial z_{0}}\left(\mathrm{D}^{\prime}\right)>0 .
\end{aligned}
$$

But, from the definition of the connection matrix, they correspond to the following:

$$
\begin{aligned}
& c_{A^{\prime} 11} * c_{B^{\prime} 11^{\prime}} c_{C^{\prime} 11} * c_{D^{\prime} 11}>0 \\
& c_{A^{\prime} 21} * c_{B^{\prime} 21^{+}} c_{C^{\prime} 21} * c_{D^{\prime} 21}>0 \\
& c_{A^{\prime} 31} * c_{B^{\prime} 31^{\prime}} c_{C^{\prime} 31} * c_{D^{\prime} 31}>0 \\
& c_{A^{\prime} 13} * c_{B^{\prime} 13^{*}} c_{C^{\prime} 13} * c_{D^{\prime} 13}>0 \\
& c_{A^{\prime} 23} * c_{B^{\prime} 23^{+}} c_{C^{\prime} 23} * c_{D^{\prime} 23}>0 \\
& c_{A^{\prime} 33} * c_{B^{\prime} 33^{*}} c_{C^{\prime} 33} * c_{D^{\prime} 33}>0
\end{aligned}
$$




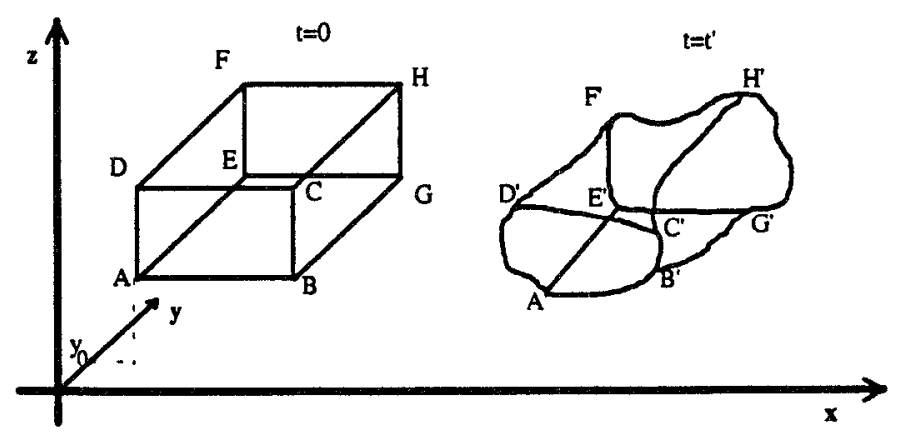

Fig. 9. Evolution of the external surface of the region of uncertainty for a third-order system.

where $c_{P_{l J}}$ is the coefficient $c_{\imath J}$ of the connection matrix in $P$. If one of these relations is not satisfied, we say that the test on connection matrices is positive, that is, the PSV does not hold.

\subsection{The Interacting Algorithm}

We have implemented an algorithm that uses the properties of the connection matrix to treat the evolution of the uncertainty region better. In this case, we do not approximate the uncertainty region with an $n$-cube at each integration step. Thus, we consider the variables as interacting for the whole simulation process.

In an $n$ th-order ODE, the external surface of the $n$-dimensional region of uncertainty can be divided into more parts, whose order is less than or equal to $n-1$. The generic part of the $i$ th order is a function of $i$ parameters. Since it is sufficient to know the evolution of the external surface, we only consider the evolution of the surface parts $S_{i}$.

For any surface $S_{i}\left(t^{*}\right)$ of $i$ th order (e.g., the line $\mathrm{D}^{\prime} \mathrm{C}^{\prime}$ of order 1 ), the algorithm computes the connection matrices for the $2^{i}$ vertices at time $t^{*}$ and compares them column by column. It considers only the $i$ columns corresponding to the $i$ parameters (e.g., only the first column, corresponding to the parameter $x_{0}$, of the connection matrices computed in $\mathrm{D}^{\prime}$ and $\mathrm{C}^{\prime}$ ). If a pair of corresponding elements of the columns have different signs, the PSV is not satisfied. Therefore, the introduction of a new trajectory is necessary (Figure 10). For symmetry, we take the trajectory starting from the geometric center of $S_{\imath}(0)$ (in this case, E, the midpoint of DC). This added initial point divides $S_{i}(0)$ into $2^{i}$ subparts (DE and $\mathrm{EC}$ ). The process can be repeated recursively for any of them.

The process is controlled by a parameter of recursion, called the sampling index, that is incremented at each recursion. The recursive process stops when it reaches the maximum value stated by the user. The sampling index may be considered as an index of the degree of roughness of the external surface of the region of uncertainty whose evolution may be simulated by our algorithm. Users may choose the accurateness of the simulation depending on their simulation demands. This procedure is repeated for any $\alpha$-cut level 


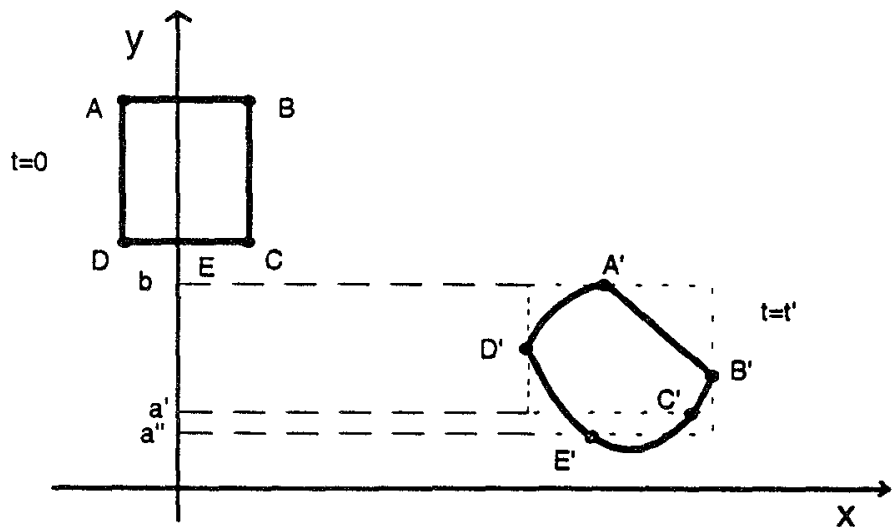

Fig. 10. $\mathrm{D}^{\prime} \mathrm{C}^{\prime}$ does not satisfy the PSV. We introduce a further trajectory, EE'.

considered in the fuzzy values discretization, thus obtaining the fuzzy evolution of the dynamic system.

\subsection{The Interacting Approach and Linear Systems}

A $n$ th-order system is linear if its dynamic behavior is represented by $\dot{x}=A x$, where $A$ is an $n$ th-order square matrix whose elements are constants. Arnol'd [1992] demonstrated that the time transformations into the phase space of linear systems map straight lines into straight lines. As a consequence, a region of uncertainty represented by a rectangle is always mapped into a quadrilateral. Therefore, it is always sufficient to know the evolution of the vertices of the rectangle in order to know its evolution. In other words, the PSV holds.

We demonstrate that the interacting approach provides always a correct solution in the presence of linear systems. Consider the evolution of the connection matrix for linear systems. Generally, the Jacobian matrix $J=$ $J(t, x)$ and the connection matrix $C=C(t, x)$ are functions of time and state variables. In the case of linear systems, $\dot{x}=A x, J$ corresponds to $A$, the matrix of coefficients of the linear system.

$J$ is a function neither of time nor of state variables. As a consequence, $C$, whose dynamic behavior is described by Eq. (17), is independent of state variables. Then $C=C(J, t)=C(t)$. At time $t^{*}$, the connection matrix is the same in any point of the phase space; the test on these matrices is always negative, and it is sufficient to consider only the trajectories of vertices. Then, we can say that in the case of linear systems the method introduces only the necessary and sufficient trajectories. This approach leads always to a correct simulation for linear systems because for these systems the PSV always holds.

\subsection{Drawbacks of the Interacting Approach}

There is a drawback to the resolution of ODEs having intervals as initial conditions, even adopting the interacting approach, due to the fact that the 


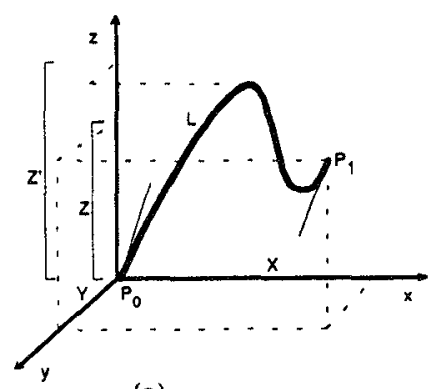

(a)

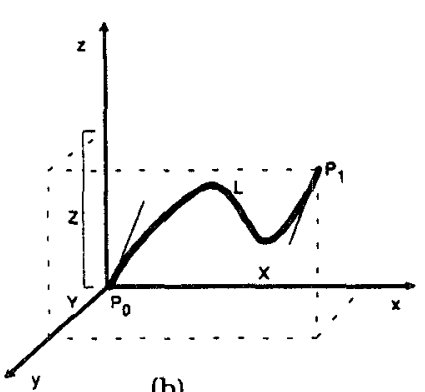

(b)

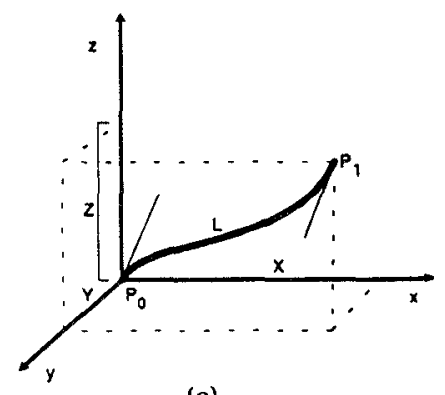

(c)

Fig. 11. Some examples of curves whose test is not positive.

test on connection matrices is a necessary but not sufficient condition for the PSV. If the test is positive, the vertices of the considered surface are not enough to determine exactly the range of state variables. The addition of another trajectory increases the precision of the simulation results. In the opposite case, when the test is not positive we cannot say anything. Consider three configurations that correspond to this situation (Figure 11). In all three cases, the test is not positive. In Figure 11(a) the extremes of line $L$ are not sufficient to compute the correct range $Z^{\prime}$ for variable $z$ (they provide $Z \subset Z^{\prime}$ ). In Figure 11(b) ( $L$ nonmonotonous) and c ( $L$ monotonous), the extremes $P_{0}$ and $P_{1}$ are sufficient to compute the correct ranges of variables $x, y$, and $z$. In Figure 11(a) the method takes into account only a limited number of points. As a result, some trajectories may not be considered. Therefore, the method produces a solution, which may be only contained in the correct one.

\section{COMPARISONS OF THE TWO APPROACHES}

The interacting approach goes a step forward as compared to the noninteracting approach. The solution of a fuzzy interacting simulation is a qualitative description of the system behavior, coherent with the real system. In general, the simulation does not introduce trajectories external to the correct evolution of the region of uncertainty, and in the case of linear models the method is optimal.

On this subject, it is interesting to compare the outputs of the two approachs when simulating the systems described in the following examples:

Example 5.1 Oscillating System.

$$
\left\{\begin{array}{l}
\dot{x}=y \\
y=-x
\end{array}\right.
$$

$x(0)$ and $y(0)$ are represented as triangular fuzzy numbers whose vertices are given, respectively, by the triples $(8,0)(9,1)(10,0)$ and $(3,0)(4,1)(5,0)$. 


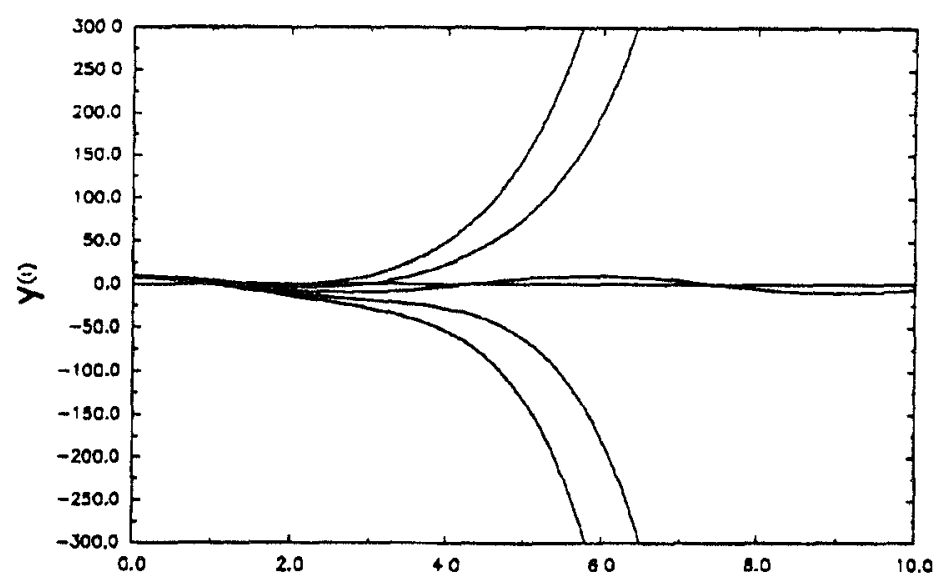

Fig. 12. Simulation with the noninteracting method. Evolution of the state variables $y(t)$.

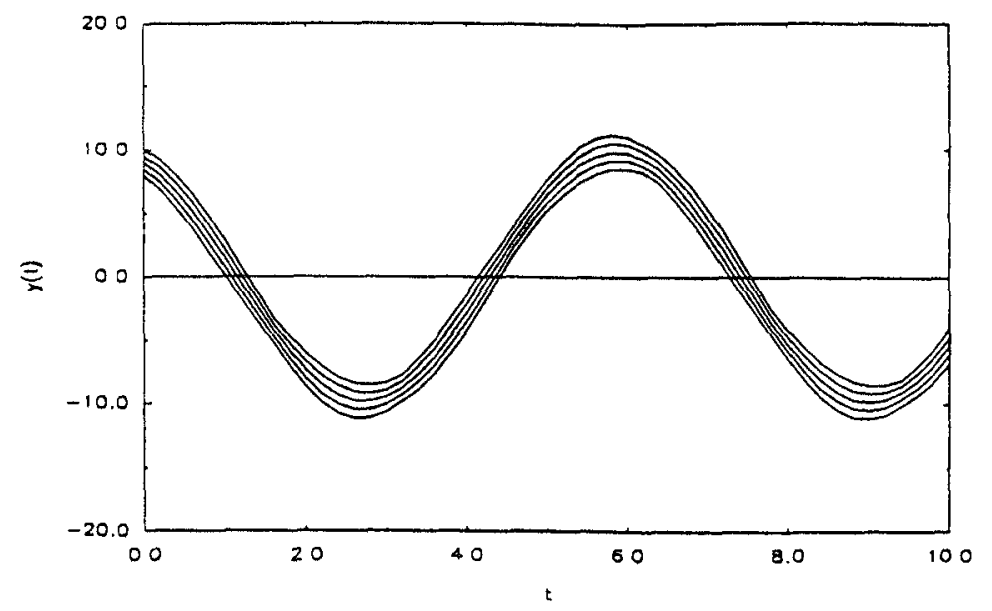

Fig, 13. Simulation with the interacting method. Evolution of the state variables $y(t)$.

After a few steps, the results of the noninteracting approach are no longer significant (Figure 12): $y(6)$ could take almost any value of its domain. The interacting approach provides the correct fuzzy description of the dynamic behavior of the system (Figure 13).

Example 5.2 Van Der Pol Equation. The Van Der Pol equation

$$
\frac{d^{2} y}{d t^{2}}+\left(Y^{2}-1\right) \frac{d y}{d t}+y=0
$$

can be expressed by the following system:

$$
\left\{\begin{array}{l}
\dot{y}=z \\
\dot{z}=-\left(y^{*} y-1\right)^{*} z-y .
\end{array}\right.
$$

ACM Transactions on Modeling and Computer Simulation, Vol. 4, No. 4, October 1994. 


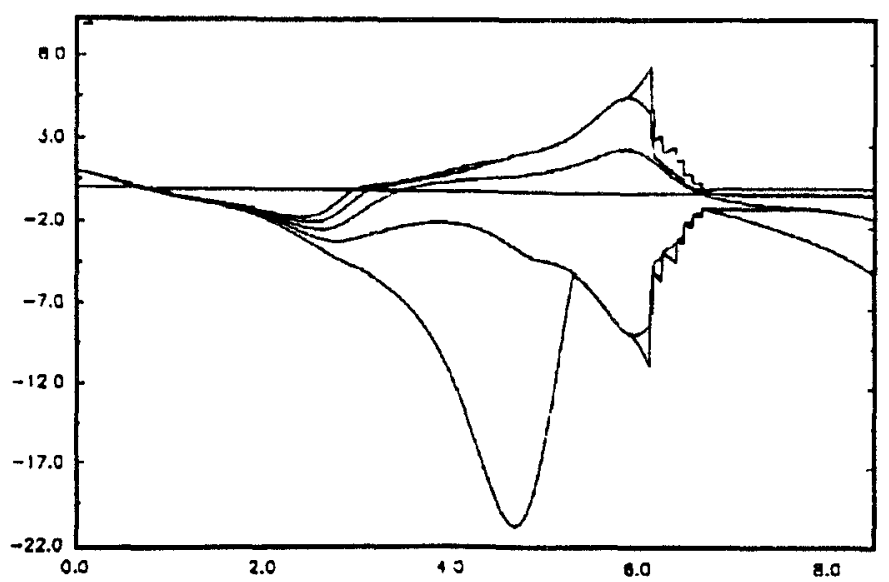

Fig. 14. Simulation with the noninteracting method. Evolution of the state variables $z(t)$.

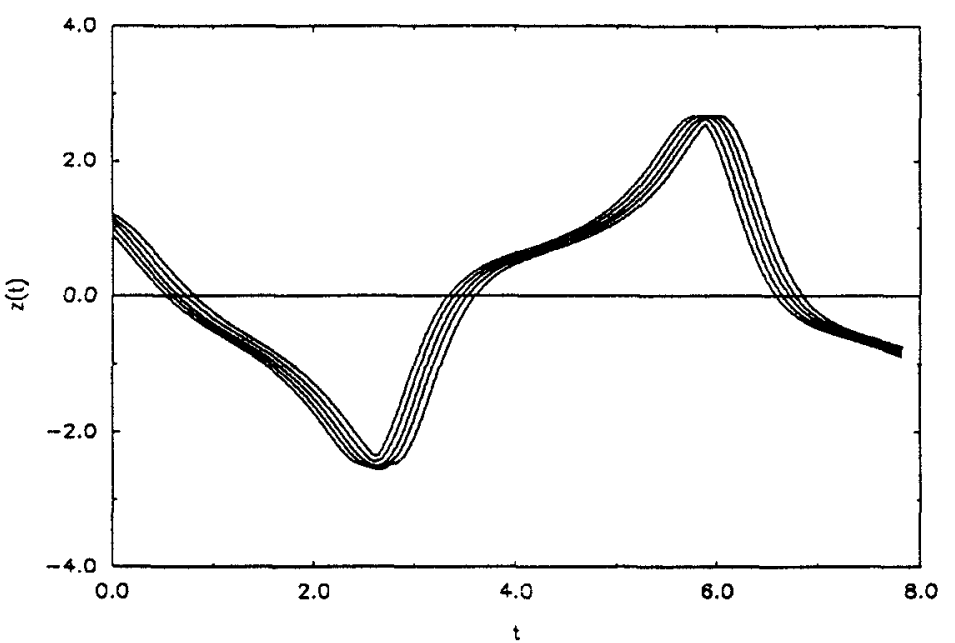

Fig. 15. Simulation with the interacting method. Evolution of the state variables $z(t)$.

$y(0)$ and $z(0)$ are represented as triangular fuzzy numbers whose vertices are given, respectively, by the triples $(0.9,0)(1,1)(1.1,0)$ and $(0.9,0)(1,1)(1.1,0)$.

In this example, too, the interacting approach provides a more correct fuzzy description of the dynamic behavior of the system (Figures 14 and 15).

\section{RELATED FUZZY SIMULATION APPROACHES}

To our knowledge, there are two proposed approaches to fuzzy simulation. The first treats mathematical models where some parameters are fuzzy (e.g., FUSIM [Shen and Leitch 1993]; Fishwick 1990]); the second is based on symbolic, qualitative reasoning and can be used when no mathematical model 
is available (e.g., Bousson and Travè-Massuyes [1993]). In this section we only discuss the first approach, since the second approach applied to a different kind of modeling.

FUSIM [Shen and Leitch 1993] extends the approach proposed in QSIM [Kuipers 1986] by considering qualitative variables that can take "fuzzy qualitative values." The transitions of a variable from a qualitative state to the next state are given by a set of rules, called the possible state transition rules. They generate all of the possible transitions from a qualitative state to its possible successors. The set of possible successors is then filtered to eliminate behaviors inconsistent with the relationships holding among variables. However, the set of next states may still contain a number of spurious behaviors. This method allows the introduction of semiquantitative information, helping the filtering activity, but this is not always available. However, the resulting envisioning tree may still be too wide to be considered useful for complex systems.

Fishwick [1990] proposed three methods for fuzzy simulation.

Monte Carlo Methods. In this case, the traditional Monte Carlo methods are used with a fuzzy distribution instead of a probability distribution. In general, these methods are appropriate when enough samples can be obtained for an application so that uncertainty can be probabilistically specified as a density distribution. However, there are applications, such as the ones cited in the introduction, where it is impossible to gather statistical information. Fishwick proposed giving fuzzy distributions as approximations of the unavailable probability distributions. However, the probabilistic sampling does not consider the dynamic properties of the system.

The correspondence between the probabilistic and the actual evolutions of the uncertainty region is not known. The points chosen by the Monte Carlo algorithm during the evolution may not be significant enough to describe the system behavior, since they have been chosen according to the initial probability density. Indeed, the probability density may change according to the dynamic evolution of the system. With Qua.Si. I, we obtain a hypersurface that includes all of the possible system trajectories. Qua.Si. II simulates those points that seem to be more significant in order to capture the variety of the system behaviors. Moreover, whereas the Monte Carlo algorithm takes a fixed number of points, Qua.Si. II can estimate the most appropriate number of points to be simulated in any situation, thus adapting the computational effort for the specific situation. The interacting approach based on the connection matrix gives a necessary condition to decide whether to select other points or not.

Correlated Method. This method assumes that all of the uncertainty sources are correlated. For instance, if we have three uncertain variables $(x, y, z)$ whose values are the triangular fuzzy numbers shown in Figure 16, numeric simulation is executed three times (one for each vertex of the triangle), assigning to the variables the numeric values $(x 1, y 1, z 1)$, $(x 2, y 2, z 2),(x 3, y 3, z 3)$ of the three vertices. Simulation is numeric, but 

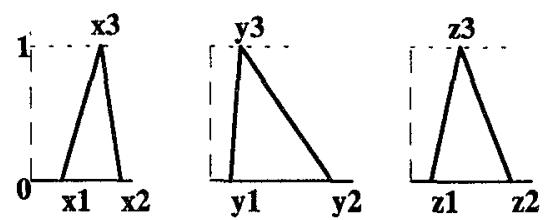

Fig. 16. Triangular fuzzy numbers.

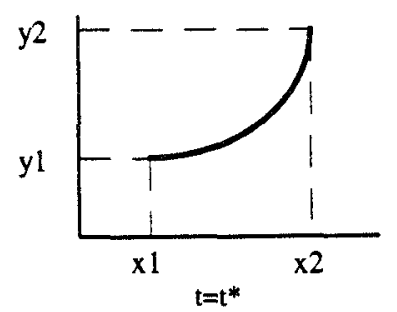

Fig. 17. Monotonous interaction.

results are still aggregated as fuzzy numbers at the end of $n$ numeric simulations.

We criticize this method for the following reasons:

(1) it is difficult to define a correlation of this type (Figure 17). In a complex system characterized by a high number of interactions and affected by uncertainty, it is not possible to state this type of simplified relation (monotonous interaction) between variables.

(2) Even if a monotonous correlation holds at a given time, we cannot guarantee that it will remain so. If variables were correlated in this way at time $t^{*}$, knowing how points $(x 1, y 1)$ and $(x 2, y 2)$ evolve does not mean that we know the relation between $x$ and $y$ at time $t^{*}+t$. Trajectories can be such that considering only the two extremes of an interval is meaningless (Figure 18).

Uncorrelated Method. This method assumes that all of the uncertainty sources are not correlated, that is, that they are independent of one another. For this purpose, Fishwick [1990] used fuzzy arithmetic in the numerical integration routine, and he annotated the divergent nature of the output, which makes that approach practically unfeasible for most applications. This is a problem, as we stressed previously, typical of any method that applies Taylor series to intervals. The consequence is a fuzzy output whose width grows at each step.

\section{SIMULATION AND FUZZY LOGIC}

In Qua.Si. I it is possible to describe the model not only by ODEs, but also by fuzzy rules. We adopt fuzzy rules to describe the aspects of a system that cannot be mathematically modeled. Moreover, a rule-based model may require less computational resources, with the same effectiveness of a model defined by heavier mathematical formalisms. 
Fig. 18. Nonmonotonous interaction.

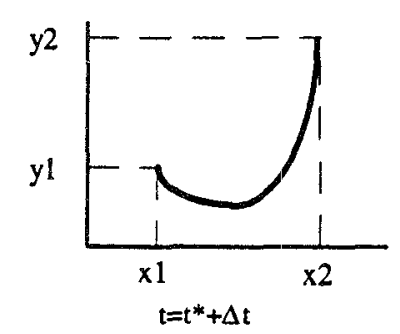

The fuzzy formalism can represent both quantitative (certain and uncertain) and qualitative knowledge. A fuzzy set can represent either a qualitative proposition or an approximate quantity. Therefore, fuzzy sets interface quantitative mathematical knowledge, represented by fuzzy mathematical formalisms, to qualitative knowledge, represented by fuzzy logic and propositions.

There are mainly two ways to identify a rule-based model:

(1) To draw it from an expert. This applies when the only source of knowledge is a human expert [Magrez and Smets 1989].

(2) To draw it from the fuzzy model of a process. There are, in fact, situations when human knowledge is narrow and inferior to modeling requirements. When a situation is complex, no expert may be able to satisfy them. In this case, we need an identification procedure to obtain the best rules for modeling the process [Sugeno and Yasukawa 1993].

Using the fuzzy formalism, we can consider any inference rule as a function with fuzzy inputs and outputs. An inference rule application may be interpreted as

(1) an input-output function, that is, the inference rule $A \rightarrow B$;

(2) a fuzzy input $X$; and

(3) a fuzzy output $Y$.

Then, it is possible to represent such a rule application as a function $Y=R_{A \rightarrow B}(X)$ with $A, B, X$ fuzzy sets. $R_{A \rightarrow B}(X)$ yields a fuzzy value $Y$, as the result of the inferential activity.

We present an example of a simple qualitative model simulated with Qua.Si. I, showing the possibilities of our approach. Suppose we want to simulate a system made of two tanks, which are filled by faucets. We want to answer the question, "What tank will be filled first? A little tank filled by two little faucets $f 1$ and $f 2$, or a large tank filled by a large flow faucet $f 3$ ?" There is a functional dependency between the opening of the faucet and the flow. We know that:

-The flow of faucet $f 1$ is proportional to the opening.

- Faucet $f 2$ is faulty and does not work till half opened; after that, its flow is equal to half of the maximum flow. 
-Faucet $f 3$ has qualitatively three types of flow, small, medium, and maximum in correspondence with one-fourth, one-half, and three-fourths of the opening.

We know, besides, that faucets are opened a little for about five seconds and then opened a lot. This description is qualitative. However, it can be modeled by a fuzzy formalism that translates the qualitative knowledge into fuzzy distributions.

We can use, for example, a qualitative inferential rule to describe the relation existing for faucet $f 3$ between the flow and the opening, and we can use fuzzy numbers to describe approximate values. At the same time, we can model by differential equations how the liquid levels rise in the tanks. Therefore, we only have qualitative knowledge, but it can be represented by a simulation model that provides an approximate description of the system. The model was implemented into the Qua.Si. I syntax and simulated qualitatively. The fuzzy evolution in time of variables $v 1$ and $v 2$ (liquid quantities into the containers) is plotted in Figure 19. Note that, although qualitative, the output can provide information that is useful for answering the initial question.

The property of treating logic rules as simple input-output functions makes it possible to integrate logic and mathematics, both being characterized by operations on the same kind of arguments: fuzzy numbers. This is possible in the noninteracting approach, where the qualitative and quantitative relations of the qualitative model share their outputs, represented as fuzzy numbers. It is not possible in the interacting approach, where the fuzzy formalism is used only as an interface between the modeler and the simulator, which works on numerical values.

\section{THE QUA.SI. SIMULATORS}

The fuzzy formalism is the unifying element of a system description that can contain both qualitative and quantitative information. Qua.Si. I implements the noninteracting approach, while Qua.Si. II implements the interacting approach. Both allow the use of many types of relations to describe a system qualitatively:

- quantitative algebraic and differential relations with numeric parameters and/or initial conditions and

- quantitative algebraic and differential relations with approximate parameters and/or initial conditions.

Only Qua.Si. I allows

-qualitative relations expressed as expert-system rules and

—qualitative relations expressed as fuzzy process laws [Sugeno 1979].

Qua.Si. I and II were implemented on a Sun Sparc workstation using the $\mathrm{C}++$ language, with an architecture based on a constraint network [Abelson and Sussmann 1985]. According to object-oriented criteria, we provided 

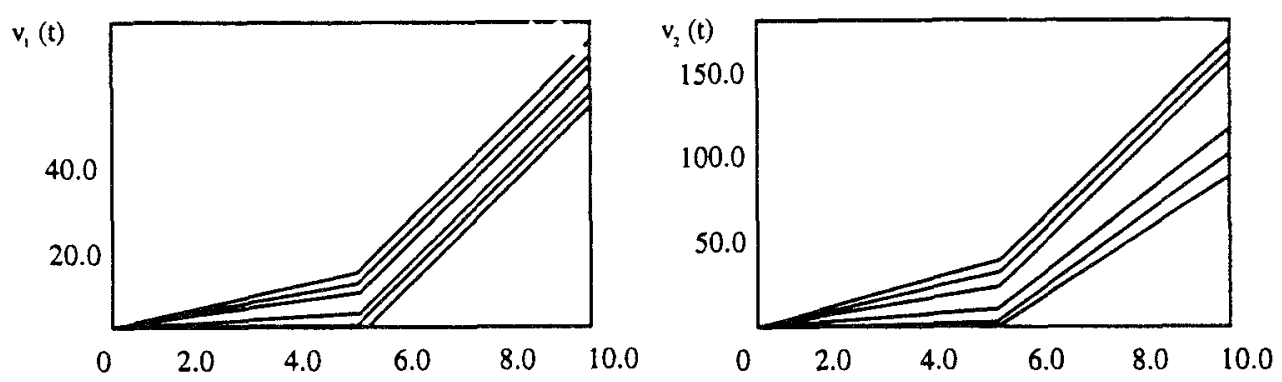

Fig. 19. Simulation with Qua.Si. I of a model with quantitative and qualitative relations. Evolution of the state variables $v 1(t)$ and $v 2(t)$ (volume of liquid in the containers).

libraries for fuzzy mathematics and logic. We applied Qua.Si. I to significant theoretical and industrial models. For instance, we simulated qualitatively a part of a nuclear power plant [Bontempi 1992].

We used Qua.Si. II to simulate theoretical models and the behavior of a part of a packaging machine. Since this application is paradigmatic for simulators like Qua.Si., we describe it here with some detail.

The device we modeled is a simple mechanical system that transforms a rotational movement into an alternative one. The aim of the device is to push some product (e.g., cigarettes, candies) into a package. The application problem consists of monitoring such a device in order to recognize the possibly dangerous behaviors, which are mainly due to some worn-out moving parts. We are implementing a knowledge-based system to do this job, with the goal of avoiding both early maintenance operations and destructive failures.

By analyzing vibrations of some parts, it is possible to obtain the so-called "derived movement law, that is, the dynamic behavior of the acceleration of the extremity of the alternately moving part, called the pusher. Unfortunately, in the present phase of the project it is not possible to collect experimental data for the situations considered dangerous, since the machines running at the client's sites cannot be stopped (to be inspected), nor are they equipped with the appropriate sensors. Moreover, there are no data about past failures, since failures are always destructive, and what remains of the device after a fault cannot give any useful numerical information about the cause. In other words, in this application, it is impossible to collect data about the situation we would like to model. Therefore, the only feasible approach consists of building a parametric model of the device correctly working (available at the factory), performing a few tests with artificially degraded parts, and giving with some approximation the parameter values for the situation of interest. This is what we did. In this case, the approximate parameters describe the degree of deterioration of the two critical moving parts. The model consists of 25 variables, among which hold relationships represented by 6 ODEs, 12 algebraic relationships, and 7 fuzzy relationships. In Figure 20 we show the results of Qua.Si. II applied to this model. On the abscissas is the time, and on the ordinates is the acceleration of the pusher.

ACM Transactions on Modeling and Computer Simulation, Vol. 4, No. 4, October 1994 


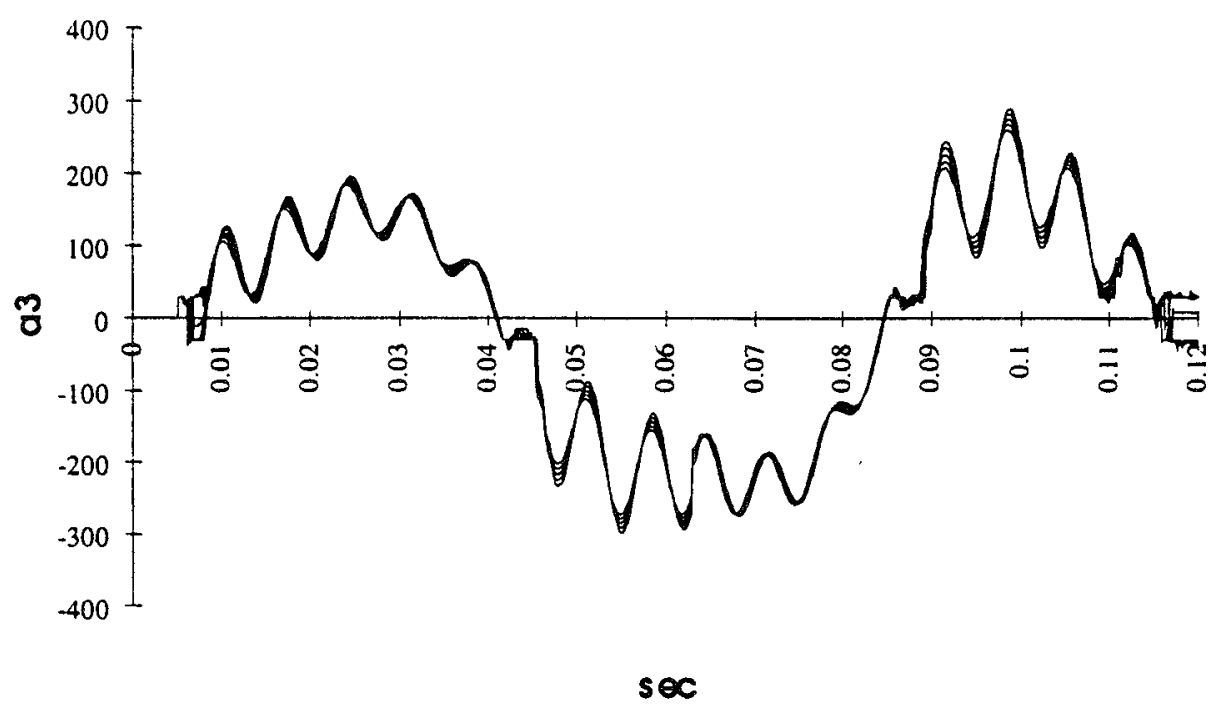

Fig. 20. Simulation with the interacting method. Evolution of the state variables $a 3$ (acceleration of the pusher).

As one can see, Qua.Si. II gives an area where the actual device behavior should stay. The derived movement law coming from the device is compared with this area. If the actual behavior can be considered coherent with the area computed with critical parameter values, then the knowledge-based system deduces that the device is reaching its critical point, and suggests that a maintenance operation be planned. On the other hand, if the actual data match the analogous area obtained with "safe" parameter values, the device is assumed to work properly.

\section{CONCLUSIONS}

We have presented a fuzzy approach, alternative to classic qualitative modeling, integrating numerical, mathematical models and qualitative, fuzzy formalisms. We consider real number mathematics as a particular case of fuzzy mathematics. This makes it possible to enrich numerical models with fuzzy values that describe the approximation present in the model. Additionally we make it possible to integrate quantitative relations and typically qualitative, descriptive methods, such as logical implication rules.

The main drawback of fuzzy simulation is that the fuzzy formalism cannot represent the interaction among variables. Every computation with fuzzy numbers must treat the arguments as noninteracting, even when they are not so. This introduces possible errors into fuzzy simulation. Then, the error introduced into the simulation may produce approximate descriptions of the system behavior that are wider than the correct ones. This article has dealt with this problem by proposing two kinds of algorithms: the noninteracting algorithm and the interacting algorithm. 
The noninteracting approach is based on the repetition of some numerical computations, thus reducing the error introduced during the integration step. The main problem of this approach is that, while the behavior of the actual system is contained in the simulated one, this may not be significant for the application. The interacting approach is based on the notion of the connection matrix and solves the most relevant problems of the noninteracting approach.

We have applied both approaches to real problems, showing how this type of qualitative, fuzzy simulation can support reasoning on device behaviors. In both of the problems we faced, it was impossible to identify the model precisely, since we had to model situations critical for the systems. Moreover, in these applications, no information about past, interesting situations was available. Therefore, we can conclude that tools like Qua.Si. I and II cover an interesting niche in the world of modeling applications.

\section{REFERENCES}

ABElson, K. AND Sussmann, P. 1985. Structure and Interpretation of Computer Programs. The MIT Press, Cambridge, Mass.

ARNOLd, C. 1992. Ordinary Differenttal Equations. Springer-Verlag, Berlin.

BAAS, S. AND KWAKERNAAK, H. 1977. Rating and ranking of multiple-aspect alternatives using fuzzy sets. Automatica 13, 47-58.

BoBrow, D., ED. 1985. Qualtative Reasoning about Physical Systems. The MTT Press, Cambridge, Mass.

Bonarini, A. AND Bontempi, G. 1994a. Qualitative simulation of approximate models: An approach based on fuzzy sets. In Cybernetics and Systems Research '94, R. Trappl, Ed. World Scientific Publications, Singapore, 359-366.

BonARINI, A. AND BontempI, G. 1994b. Qua. Si.: A qualitative simulation approach for fuzzy models. In Modeling and Simulation ESM 94 (Proceedings European Simulation Multiconference 1994, A. Guasch and R. M. Huber, Eds. SCS International, Ghent, Belgium, 420-424.

BONTEMPI, G. 1993. Simulation under uncertainty: An approach based on fuzzy sets. Laurea thesis, Politecnico di Milano, Milano (in Italian).

Bousson, K. And Travi-Massuyes. 1993. Fuzzy causal simulation in process engineering. In Proceedings of IJCAI 1993. Morgan Kaufmann, San Mateo, Calif.

Dong, W. AND SHAH, H. C. 1987. Vertex method for computing functions of fuzzy variables. Fuzzy Sets Syst. 24, 1, 65-78.

Dubors, D. And Prade, H. 1980. Fuzzy Sets and Systems. Academic Press, New York.

Dubois, D. And PRADE, H. 1979. Fuzzy real algebra: Some results. Fuzzy Sets Syst. 2, 4, 327-348.

EYKHOFF, P. 1974. System Identification-Parameter and State Estimation. Wiley, New York.

Fishwick, P. A. 1990. Fuzzy simulation: Specifying and identifying qualitative models. Int. J. Gen. Syst. 19, 2, 295-316.

GRAUPE, D. 1972. Identification of Systems. Van Nostrand Reinhold, New York.

KANDEL, A. 1986. Fuzzy Mathematical Techniques with Applications. Addison-Wesley, Reading, Mass.

KaUfmann, A. ANd GuPTA, M. 1985. Introduction to Fuzzy Arithmetlc: Theory and Applicatzons. Van Nostrand Reinhold, New York.

KUIPERS, B. 1986. Qualitative simulation. Artlf. Intell. 29, 12, 289-338.

Magrez, P. AND Suets, P. 1989. Fuzzy modus ponens: A new model suitable for applications in knowledge-based systems. Int. J. Intell. Syst. 4, 11, 181-200. (Reprinted in Readings in Fuzzy Sets for Intelligent Systems, D. Dubois, H. Prade, and R. R. Yager, Eds., Morgan Kaufmann, San Mateo, Calif, 1993, 565-574.

ACM Transactions on Modeling and Computer Simulation, Vol 4, No. 4, October 1994. 
MILNE, R. 1991. Second generation expert systems: The application gap. In Proceedings of the 11th International Conference on Expert Systems and Their Applications (General Conference on 2nd Generation Expert Systems) (Avignon, France). EC2, Nanterre, France, 259-264.

Moore, R. E. 1966. Interval Analysis. Prentice-Hall, Englewood Cliffs, N.J.

Nguyen, H. T. 1978. A note on the extension principle for fuzzy sets. J. Math. Anal. Appl.64, $2,369-380$.

Shen, Q. AND LeItch, R. 1993. Fuzzy qualitative simulation. IEEE Trans. Syst. Man Cybernet. 23,4 .

SugENo, M. 1979. Application of fuzzy sets and logic to control: A survey. J. Soc. Instrum. Cont. Eng. 18, 2.

Sugeno, M. AND Yasukawa, T. 1993. A fuzzy-logic-based approach to qualitative modeling. IEEE Trans. Fuzzy Syst. 1 1, 7-31.

WELD, D. AND DE KLEER, J. 1990. In Readings in Qualitative Reasoning about Physical Systems, D. Weld and J. De Kleer, Eds., Morgan-Kaufmann, San Mateo, Calif.

ZADEH, L. A. 1965. Fuzzy sets. Inf. Control 8, 338-353.

Zeiglen, B. P. 1976. Theory of Modelling and Simulation. Wiley, New York.

Received January 1994; revised August 1994; accepted November 1994 